Intake of alpha-linolenic acid is not consistently associated with a lower risk of peripheral artery disease results from a Danish cohort study

Bork, Christian S.; Lasota, Anne N.; Lundbye-Christensen, Søren; Jakobsen, Marianne Uhre; Tjønneland, Anne; Calder, Philip C.; Schmidt, Erik B.; Overvad, Kim

Published in:

British Journal of Nutrition

DOI:

10.1017/S0007114519000874

Publication date:

2019

Document version

Publisher's PDF, also known as Version of record

Citation for published version (APA):

Bork, C. S., Lasota, A. N., Lundbye-Christensen, S., Jakobsen, M. U., Tjønneland, A., Calder, P. C., Schmidt, E. B., \& Overvad, K. (2019). Intake of alpha-linolenic acid is not consistently associated with a lower risk of peripheral artery disease: results from a Danish cohort study. British Journal of Nutrition, 122(1), 86-92. https://doi.org/10.1017/S0007114519000874 


\title{
Intake of $\alpha$-linolenic acid is not consistently associated with a lower risk of peripheral artery disease: results from a Danish cohort study
}

\author{
Christian S. Bork ${ }^{1,2 *}$, Anne N. Lasota ${ }^{3,4}$, Søren Lundbye-Christensen ${ }^{5}$, Marianne U. Jakobsen ${ }^{6}$, \\ Anne Tjønneland ${ }^{7,8}$, Philip C. Calder ${ }^{2,9}$, Erik B. Schmidt ${ }^{1,4}$ and Kim Overvad ${ }^{1,10}$ \\ ${ }^{1}$ Department of Cardiology, Aalborg University Hospital, Hobrovej 18-22, 9000 Aalborg, Denmark \\ ${ }^{2}$ Human Development \& Health, Faculty of Medicine, University of Southampton, MP887 Southampton General Hospital, \\ Tremona Road, Southampton SO16 6YD, UK \\ ${ }^{3}$ Department of Vascular Surgery, Aalborg University Hospital, Hobrovej 18-22, 9000 Aalborg, Denmark \\ ${ }^{4}$ Department of Clinical Medicine, Aalborg University, Soendre Skovvej 15, 9000 Aalborg, Denmark \\ ${ }^{5}$ Unit of Clinical Biostatistics, Aalborg University Hospital, Soendre Skovvej 15, Aalborg, Denmark \\ ${ }^{6}$ Division of Diet, Disease Prevention and Toxicology, National Food Institute, Technical University of Denmark, Kemitorvet, \\ 2800 Kgs. Lyngby, Denmark \\ ${ }^{7}$ Danish Cancer Society Research Center, Strandboulevarden 49, 2100 Copenhagen, Denmark \\ ${ }^{8}$ Department of Public Health, Faculty of Health and Medical Sciences, University of Copenhagen, Copenhagen, Denmark \\ ${ }^{9}$ NIHR Southampton Biomedical Research Centre, University Hospital Southampton NHS Foundation Trust and University of \\ Southampton, Tremona Road, Southampton SO16 6YD, UK \\ ${ }^{10}$ Department of Public Health, Aarhus University, Bartholins Allé 2, 8000 Aarhus, Denmark
}

(Submitted 11 September 2018 - Final revision received 8 March 2019 - Accepted 25 March 2019 - First published online 20 June 2019)

\section{Abstract}

Intake of the plant-derived $n$ - 3 fatty acid $\alpha$-linolenic acid (ALA) has been associated with anti-atherosclerotic properties. However, information on the association between ALA intake and development of peripheral artery disease (PAD) is lacking. In this follow-up study, we investigated the association between dietary intake of ALA and the rate of PAD among middle-aged Danish men and women enrolled into the Danish Diet, Cancer and Health cohort between 1993 and 1997. Incident PAD cases were identified through the Danish National Patient Register. Intake of ALA was assessed using a validated FFQ. Statistical analyses were performed using Cox proportional hazard regression allowing for separate baseline hazards among sexes and adjusted for established risk factors for PAD. During a median of 13.6 years of follow-up, we identified 950 valid cases of PAD with complete information on covariates. The median energy-adjusted ALA intake within the cohort was $1.76 \mathrm{~g} / \mathrm{d}(95 \%$ central range: $0 \cdot 94-3 \cdot 28)$. In multivariable analyses, we found no statistically significant association between intake of ALA and the rate of PAD $(P=0.339)$. Also, no statistically significant associations were observed in analyses including additional adjustment for co-morbidities and in sex-specific analyses. In supplemental analyses with additional adjustment for potential dietary risk factors, we found a weak inverse association of PAD with ALA intake above the median, but the association was not statistically significant $(P=0 \cdot 314)$. In conclusion, dietary intake of ALA was not consistently associated with decreased risk of PAD.

Key words: $\alpha$-Linolenic acid: $n$-3 Fatty acids: Peripheral artery disease: Cohort studies

Peripheral artery disease (PAD) in the lower extremities is a chronic atherosclerotic disease characterised by stenosis and occlusion of the arteries, covering a clinical spectrum from no symptoms to effort-induced ischaemic muscle discomfort and/or pain and critical ischaemia with tissue $\operatorname{loss}^{(1-3)}$. Symptomatic PAD is associated with functional limitations, diminished quality of life and a high risk for major cardiovascular events and death $^{(4,5)}$. The global burden of PAD is expected to increase markedly in the near future and identification of factors that may lower disease risk is urged $^{(6)}$.

The plant-derived $n-3$ fatty acid $\alpha$-linolenic acid (ALA) is a precursor of long-chain (LC) $n-3$ PUFA, which may influence inflammatory processes that may be involved in development and progression of atherosclerosis ${ }^{(7-9)}$. However, the conversion

Abbreviations: ALA, $\alpha$-linolenic acid; LC, long-chain; PAD, peripheral artery disease.

* Corresponding author: C. S. Bork, email c.bork@rn.dk 
capacity of ALA into LC $n$ - 3 PUFA may be limited in humans ${ }^{(10)}$. ALA may possibly exert health benefits independent of its precursor role ${ }^{(11)}$ and has been suggested to be an important nutrient in the Mediterranean $\operatorname{diet}^{(12)}$, which has ascribed many health benefits including lowering risk of $\mathrm{PAD}^{(13)}$ and major cardiovascular events ${ }^{(14)}$.

The majority of previous follow-up studies investigating the association between ALA intake and the risk of atherosclerotic CVD have focused on CHD. Some cohort studies have reported inverse associations between ALA intake and CHD risk ${ }^{(15-20)}$, but the results were not consistent ${ }^{(18,21-27)}$. Few studies have investigated associations between ALA intake and the risk of ischaemic stroke ${ }^{(22,23,28-30)}$, but to our knowledge no previous follow-up studies have investigated the association between ALA intake and the risk of PAD.

The objective of the present study was to investigate the association between intake of ALA and the risk of PAD. We hypothesised that intake of ALA would be inversely associated with the risk of incident PAD.

\section{Methods}

\section{Study population and design}

This follow-up study was based on data from the Diet, Cancer and Health cohort that was established to investigate the role of diet and lifestyle in relation to cancer and other chronic diseases $^{(31)}$. The recruitment procedures, sample size considerations and collection of data have been described in detail elsewhere ${ }^{(31)}$. Briefly, native citizens aged 50-64 years who were living in and around Copenhagen and Aarhus in Denmark without a previous diagnosis of cancer were invited between 1993 and 1997 to participate in the study. Potential eligible participants were identified through the Danish Civil Registration System in which every citizen living in Denmark is provided with a unique identification number ${ }^{(31)}$.

The Diet, Cancer and Health cohort study was conducted according to the guidelines laid down in the Declaration of Helsinki and approved by the Health Research Ethics, Capital Region of Denmark, and the Danish Data Protection Agency. All participants gave written informed consent at inclusion ${ }^{(31)}$.

In the present follow-up study, participants registered with a diagnosis of cancer not registered in the Danish Cancer Registry at the time of invitation due to processing delay as well as participants registered with a diagnosis of PAD or chronic kidney disease before enrolment were excluded. Also, participants for whom information on exposure and/or covariates was missing were excluded. The current study has been approved by the Danish Data Protection Agency (2008-58-0028-2016-229).

\section{Exposure assessment}

Information on habitual diet over the past 12 months was assessed at baseline using a 192-item semi-quantitative $\mathrm{FFQ}^{(32)}$. The average consumption of foods and beverages was reported within twelve categories ranging from never to eight or more times per day. The reported intakes were used to estimate daily intake of ALA and total energy based on Danish food composition tables (version 1.3.2) with the use of the software program
FoodCalc (Center for Applied Computer Science, University of Copenhagen; www.ibt.ku.dk/jesper/foodcalc). The FFQ used has previously been validated against two 7-d diet weighed records and found useful for categorising individuals according to their intake of energy and polyunsaturated fat ${ }^{(33)}$. The intake of ALA was expressed as energy-adjusted intake in $\mathrm{g} / \mathrm{d}$ using the residual method ${ }^{(34)}$.

\section{Covariates}

Detailed information on social, lifestyle and health aspects such as length of schooling, smoking habits, physical activity, history of hypercholesterolaemia, hypertension and diabetes, and use of lipid-lowering or anti-hypertensive agents and insulin were collected at baseline using a self-administered questionnaire. The questionnaire was processed by optical scanning and checked for reading errors and omissions at the two study centres where anthropometric measurements including height, weight and waist circumference were also obtained at enrolment. Information on alcohol consumption, total energy intake and intake of other nutrients were obtained from the FFQ.

\section{Outcome assessment}

Incident cases of PAD were identified through record linkage with the Danish National Patient Register, which includes information on outpatient visits and discharge diagnoses from all hospitals in Denmark ${ }^{(35,36)}$.

Potential cases of PAD included participants registered with either a primary or secondary discharge diagnosis of PAD according to the International Classification of Diseases codes (International Classification of Diseases-8: 44390; 44500; 44509; 44590; 44599; 44020 and 44030, and International Classification of Diseases-10: I70.2, I70.2A and I73.9A-C). Subsequently, all potential cases of PAD were validated by review of medical records ${ }^{(37)}$. A registered diagnosis of PAD was considered as valid in patients with an ankle brachial index $<0 \cdot 9$, a toe brachial index $<0.7$ and/or demonstration of radiologically significant stenosis or calcifications in relevant arteries in the lower extremities. Patients with an ankle brachial index $>1.4$ together with a history of diabetes mellitus or treatment by chronic renal dialysis were also considered valid PAD cases. Furthermore, patients who experienced a blood pressure drop in the lower extremities of more than $30 \mathrm{mmHg}$ or a $20 \%$ drop of the resting ankle brachial index immediately after a treadmill test and patients who underwent relevant vascular surgery for atherosclerosis were included as cases. Finally, patients who qualified for vascular surgery for atherosclerosis although not performed due to severe comorbidity were also included as cases ${ }^{(37)}$.

Participants were followed up from baseline until first registration of $\mathrm{PAD}$, death, emigration or end of follow-up in December 2009.

\section{Statistics}

Hazard ratios were used to describe associations between energy-adjusted ALA intake and the rate of PAD. Hazard ratios with $95 \%$ CI were calculated using Cox proportional hazard regression with age as underlying timescale allowing for separate baseline hazards among men and women. 


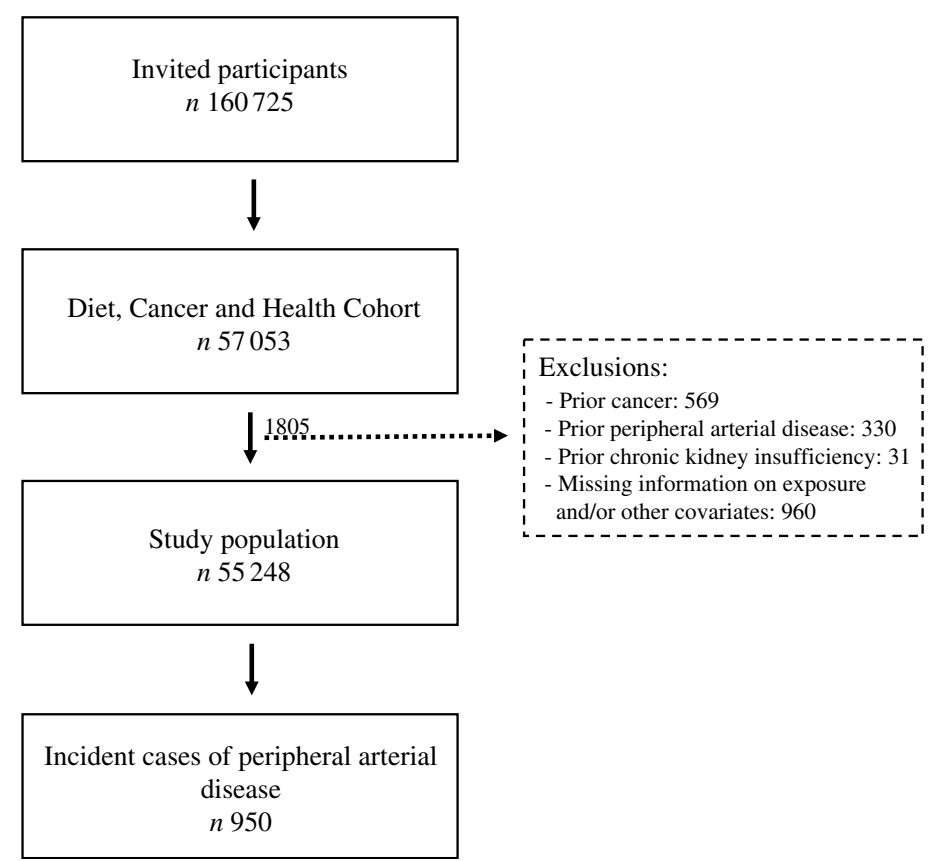

Fig. 1. Flowchart of participants in the Diet, Cancer and Health cohort and incident cases of peripheral artery disease identified during follow-up.

Dietary intake of ALA was analysed as a continuous exposure variable using restricted cubic splines with three knots with the median intake as reference in order to visualise the shape of the observed associations. The knots were placed at the 10th, 50th and 90 th percentile as recommended by Harrell( ${ }^{(38)}$. The spline curves were formally tested against a horizontal line using Wald tests. The spline plots were restricted to the $95 \%$ central range and presented with $95 \% \mathrm{CI}$. Also, analyses with ALA intake divided into quintiles were conducted using the lowest quintile as reference.

We investigated the association between ALA intake and rate of PAD in three different models specified prior to data analysis. In model 1A, we included baseline age (continuous, years) in order to ensure comparison of participants for whom reported exposure information was of the same age. In model 1B, we additionally included information on established risk factors for PAD including length of schooling ( $\leq 7,8-10$ or $>10$ years), smoking (never, former, current 1-14, 15-24 or $>24 \mathrm{~g} / \mathrm{d}$ ), physical activity (inactive, moderately inactive, moderately active or active), waist-circumference (continuous, $\mathrm{cm}$ ), BMI (continuous, $\mathrm{kg} / \mathrm{m}^{2}$ ) and alcohol intake (continuous, $\mathrm{g} / \mathrm{d}$ ). In model 2, we included the following co-morbidities, which may be considered potential intermediate variables: self-reported history of hypercholesterolaemia and/or use of lipid-lowering medication (yes, no or unknown), hypertension and/or use of antihypertensive medication (yes, no or unknown) and diabetes mellitus and/or use of insulin.

The proportional hazard assumption was evaluated by plotting the scaled Schoenfeld residuals against age at event.

In sensitivity analyses, we plotted the whole exposure range and examined whether the spline curves were robust when the number and location of the knots were modified. Also, additional adjustment for a history of myocardial infarction, ischaemic stroke or both prior to enrolment was undertaken as sensitivity analyses.
In supplemental analyses, we investigated the association between ALA intake and the rate of PAD in sex-specific analyses. Also, analyses including adjustment for established risk factors (model 1B) and hormone substitution were undertaken. In further supplemental analyses, potential dietary risk factors were added to model $1 \mathrm{~B}$ including total energy intake without contribution from alcohol intake (continuous, $\mathrm{kJ} / \mathrm{d}$ ), intake of fibre (continuous, g/d), glycaemic load (continuous, unit-less), and intakes of SFA, MUFA, linoleic acid and marine LC $n-3$ PUFA (continuous, g/d) (model 3). All continuous covariates were entered into the models using restricted cubic splines with five knots.

Data were analysed using Stata statistical software (version 15; StataCorp LP), and a $P$ value $<0.05$ was considered statistically significant.

\section{Results}

A total of 160725 men and women were invited to participate in the Diet Cancer and Health cohort study and 57053 accepted. We excluded 1805 participants because they had a diagnosis of cancer ( $n$ 569), PAD ( $n$ 330) or chronic kidney disease ( $n$ 31) before enrolment, or for whom information on exposure or other covariates was missing ( $n$ 960) (Fig. 1). Among the remaining 55248 participants, we identified 950 participants who developed PAD during a median of 13.6 (95\% central range: $4 \cdot 3-15 \cdot 3$ ) years of follow-up. The incidence rate of PAD was 1.32 per 1000 person years.

Baseline characteristics of the participants with complete information on covariates in the cohort and participants who developed PAD during follow-up are presented in Table 1.

The median energy-adjusted dietary ALA intake in the cohort was $1.76 \mathrm{~g} / \mathrm{d}$ (95\% central range: $0.94-3.28)$. 
Table 1. Baseline characteristics

(Medians and 2.5th, 97.5th percentiles; percentages)

\begin{tabular}{|c|c|c|c|c|}
\hline & \multicolumn{2}{|c|}{ Cohort (n 55248 ) } & \multicolumn{2}{|c|}{ PAD cases $(n 950)$} \\
\hline & Median & $\begin{array}{l}2 \cdot 5 \text { th, } 97.5 \text { th } \\
\text { percentiles }\end{array}$ & Median & $\begin{array}{l}\text { 2.5th, } 97.5 \text { th } \\
\text { percentiles }\end{array}$ \\
\hline \multicolumn{5}{|l|}{$\operatorname{Sex}(\%)$} \\
\hline Males & & $47 \cdot 7$ & & $62 \cdot 1$ \\
\hline Females & & $52 \cdot 3$ & & 37.9 \\
\hline $\begin{array}{l}\text { Age at enrolment } \\
\text { (years) }\end{array}$ & $56 \cdot 1$ & $50 \cdot 5,64 \cdot 7$ & $58 \cdot 6$ & $50 \cdot 7,64 \cdot 9$ \\
\hline \multicolumn{5}{|l|}{ Length of schooling (\%) } \\
\hline$\leq 7$ years & & $32 \cdot 7$ & & $48 \cdot 0$ \\
\hline $8-10$ years & & $46 \cdot 2$ & & $40 \cdot 2$ \\
\hline$>10$ years & & $21 \cdot 1$ & & $11 \cdot 8$ \\
\hline \multicolumn{5}{|l|}{ Smoking (\%) } \\
\hline Never & & 35.4 & & 4.8 \\
\hline Former & & $28 \cdot 9$ & & $18 \cdot 0$ \\
\hline Current $<15 \mathrm{~g} / \mathrm{d}$ & & $13 \cdot 0$ & & $19 \cdot 0$ \\
\hline Current $15-25 \mathrm{~g} / \mathrm{d}$ & & $16 \cdot 0$ & & $40 \cdot 4$ \\
\hline Current $>25 \mathrm{~g} / \mathrm{d}$ & & $6 \cdot 8$ & & $17 \cdot 7$ \\
\hline \multicolumn{5}{|l|}{ Physical activity (\%) } \\
\hline Inactive & & $10 \cdot 8$ & & $16 \cdot 3$ \\
\hline Moderately inactive & & $30 \cdot 4$ & & $32 \cdot 0$ \\
\hline Moderately active & & $24 \cdot 2$ & & $21 \cdot 2$ \\
\hline Active & & 34.7 & & 30.5 \\
\hline $\begin{array}{l}\text { Waist circumference } \\
(\mathrm{cm})\end{array}$ & $89 \cdot 0$ & $67 \cdot 0,115 \cdot 0$ & $91 \cdot 3$ & $68 \cdot 8,117 \cdot 0$ \\
\hline BMI $\left(\mathrm{kg} / \mathrm{m}^{2}\right)$ & $25 \cdot 5$ & $19 \cdot 6,35 \cdot 7$ & $25 \cdot 5$ & $19 \cdot 3,34 \cdot 7$ \\
\hline Alcohol intake (g/d) & $12 \cdot 9$ & $0 \cdot 2,81 \cdot 0$ & $16 \cdot 5$ & $0 \cdot 0,104 \cdot 7$ \\
\hline \multicolumn{5}{|l|}{ Co-morbidities (\%) } \\
\hline Hypercholesterolaemia & & 7.4 & & $13 \cdot 8$ \\
\hline Hypertension & & $16 \cdot 1$ & & $27 \cdot 8$ \\
\hline Diabetes mellitus & & $2 \cdot 0$ & & $10 \cdot 7$ \\
\hline \multicolumn{5}{|l|}{ Dietary factors } \\
\hline $\begin{array}{l}\text { Total energy intake } \\
\text { (kJ) }\end{array}$ & $8895 \cdot 2$ & $\begin{array}{l}4937 \cdot 2 \\
15142 \cdot 9\end{array}$ & $8749 \cdot 6$ & $\begin{array}{l}4730 \cdot 1 \\
15537 \cdot 0\end{array}$ \\
\hline Intake of fibre ${ }^{\star}$ & $20 \cdot 6$ & $12 \cdot 3,31 \cdot 9$ & $19 \cdot 3$ & $11 \cdot 1,29 \cdot 9)$ \\
\hline Glycaemic load* & $189 \cdot 8$ & $98 \cdot 8,333 \cdot 3$ & $182 \cdot 7$ & $93.5,335.9$ \\
\hline SFA ${ }^{*}$ & $28 \cdot 7$ & $16 \cdot 4,42 \cdot 4$ & 31.4 & $18 \cdot 5,44 \cdot 2$ \\
\hline MUFA* & $28 \cdot 1$ & $16 \cdot 3,43 \cdot 3$ & 31.3 & $18 \cdot 3,46 \cdot 0$ \\
\hline Linoleic acid* & $11 \cdot 0$ & $5 \cdot 7,20 \cdot 1$ & 11.4 & $6 \cdot 2,20 \cdot 0$ \\
\hline Long-chain $n$-3 PUFA* & 0.7 & $0.1,1 \cdot 7$ & 0.7 & $0 \cdot 2,1 \cdot 8$ \\
\hline$\alpha$-Linolenic acid* & 1.8 & $0.9,3.3$ & 1.9 & $1.0,3.4$ \\
\hline
\end{tabular}

PAD, peripheral artery disease.

* Energy-adjusted intake.

In spline analyses including adjustment for sex and age (model 1A), we found a positive association between ALA intake and the rate of PAD (online Supplementary Fig. S1). However, in multivariable analyses including additional adjustment for established risk factors for PAD (model 1B) a weak statistically non-significant inverse U-shaped association between ALA intake and the rate of PAD was observed $(P=0 \cdot 339)$ (Fig. 2). Additional adjustment for co-morbidities (model 2) also showed a weak inverse U-shaped association between ALA intake and the rate of PAD that was not statistically significant $(P=0.338)$ (online Supplementary Fig. S2). In supplemental analyses, with additional adjustment for dietary risk factors (model 3), we found a weak inverse association to PAD above the median ALA intake, but the overall association was not statistically significant different from a horizontal line $(P=0 \cdot 314)$ (online Supplementary Fig. S3).

Sensitivity analyses indicated that models with ALA intake modelled as restricted cubic splines were robust when the location and number of knots for the exposure of interest were modified.
Categorical analyses of the association between ALA intake in quintiles and the rate of PAD are presented in Table 2. We found similar patterns of associations in analyses of ALA intake in quintiles and the rate of PAD as in the spline analyses. The individual hazards in the second to fifth quintile were not statistically significantly different from the reference in the first quintile in either of the multivariable adjusted models. In supplemental analyses, additional adjustment for myocardial infarction and/or ischaemic stroke before enrolment did not influence the observed associations. Also, additional adjustment for use of hormone substitution did not influence the observed associations (data not shown).

Similar patterns of associations between ALA intake and the rate of PAD were observed when the analyses were conducted separately among men and women (online Supplementary Table S2).

No evidence of a departure from the proportionality assumption was observed in either of the models (data not shown).

\section{Discussion}

In this large follow-up study, we found indications of a weak inverse U-shaped association between ALA intake and the rate of PAD in analyses including adjustment for established risk factors and indications of a weak inverse association between ALA intake and the rate of PAD above the median intake in analyses including adjustment for established risk factors and dietary risk factors. However, none of these associations was statistically significant. Given the relatively weak and statistically nonsignificant associations, the present study suggests that dietary intake of ALA is not appreciably associated with the risk of PAD within this population of middle-aged Danish men and women. It should be stressed that the present study did not investigate the potential effect of a Mediterranean diet on PAD risk, but the results may indicate that the possible protective effect provided by the Mediterranean dietary pattern on PAD and major cardiovascular events is unlikely to be ascribed to ALA intake.

The present study had some limitations that should be mentioned. Participants were followed by linkage with nationwide registries with very limited loss to follow-up, which limits the potential of selection bias. Information on ALA intake was obtained using a self-administered FFQ and measurement error is inevitable, but because of the temporality in a follow-up study, exposure measurement error probably occurred at random, which generally leads to an underestimation of the true association and loss of statistical power. The FFQ used in the present study was not specifically developed to assess ALA intake. Further, information on diet was only available at baseline and changes in dietary habits during follow-up may have occurred. Thus, repeated dietary measurements would have been preferable to limit random measurement error and to capture potential changes in dietary habits over time. However, diets of individuals tend to be relatively consistent over intervals of several years ${ }^{(39)}$. Information bias is unlikely to have influenced the observed associations significantly because 


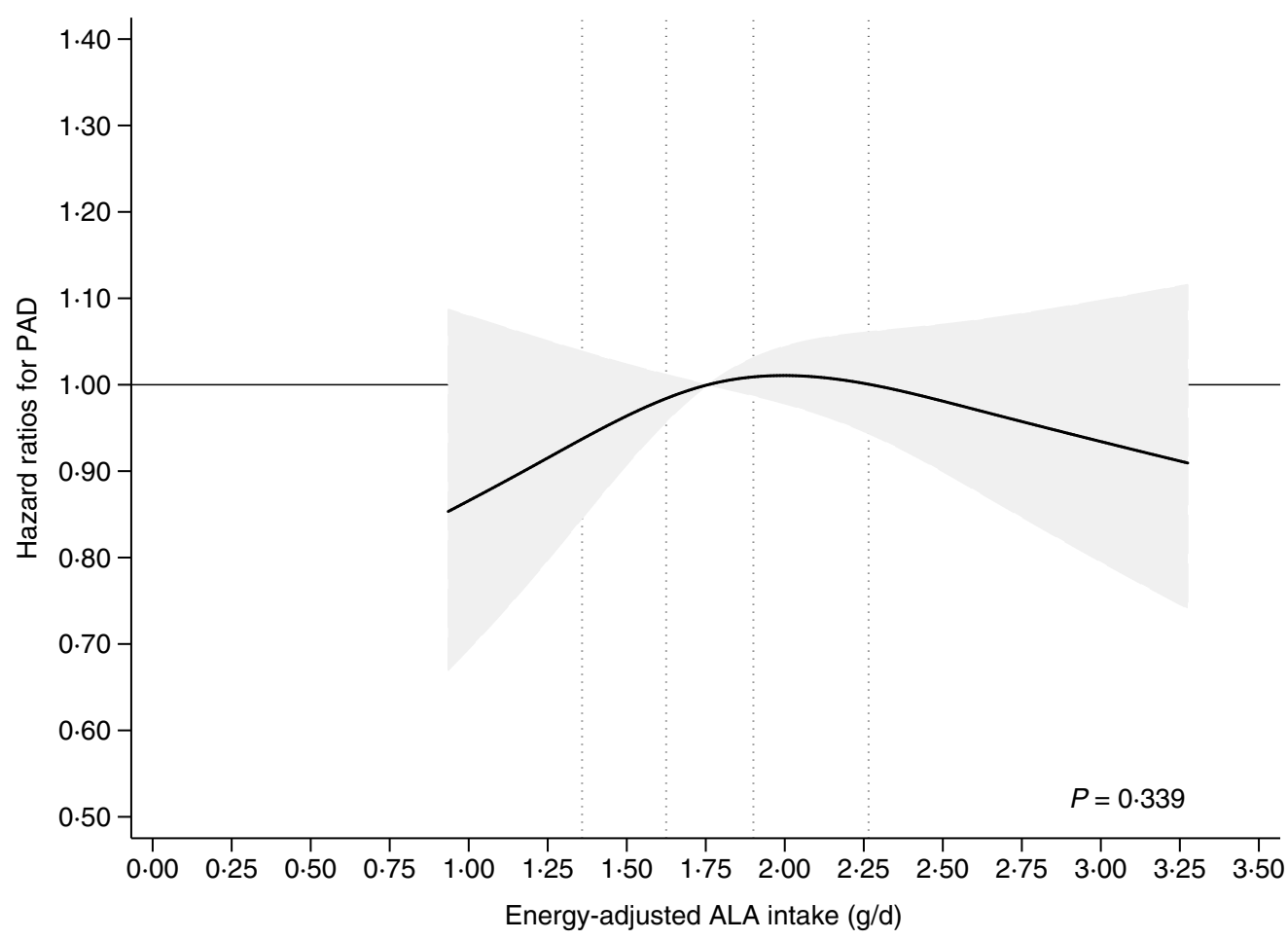

Fig. 2. Intake of $\alpha$-linolenic acid (ALA) and the risk of incident peripheral artery disease (PAD). The multivariable analyses were conducted using Cox proportional hazard regression including adjustment for established PAD risk factors (model 1B) with the median intake of ALA as reference. The 20th, 40th, 60th and 80th percentiles of ALA intake are shown with dotted lines. The shaded grey area indicates the $95 \% \mathrm{Cl}$ of hazard ratios of PAD — . The spline plot is shown for the $2.5-97.5$ percentiles of ALA intake.

Table 2. Quintiles of energy-adjusted $\alpha$-linolenic acid (ALA) intake and risk for peripheral artery disease* (Hazard ratios (HR) and $95 \%$ confidence intervals)

\begin{tabular}{|c|c|c|c|c|c|c|c|c|c|}
\hline \multirow{2}{*}{$\begin{array}{l}\text { Quintiles of ALA } \\
\text { intake }\end{array}$} & \multirow{2}{*}{$\begin{array}{c}\text { Cases } \\
(n)\end{array}$} & \multicolumn{2}{|c|}{ Model $1 \mathrm{~A} \dagger$} & \multicolumn{2}{|c|}{ Model 1B $\ddagger$} & \multicolumn{2}{|c|}{ Model $2 \S$} & \multicolumn{2}{|c|}{ Model 3\| } \\
\hline & & $\mathrm{HR}$ & $95 \% \mathrm{Cl}$ & $\mathrm{HR}$ & $95 \% \mathrm{Cl}$ & $\mathrm{HR}$ & $95 \% \mathrm{Cl}$ & $\mathrm{HR}$ & $95 \% \mathrm{Cl}$ \\
\hline$<1.36 \mathrm{~g} / \mathrm{d}$ & 116 & 1 & Reference & 1 & Reference & 1 & Reference & 1 & Reference \\
\hline $1.36-1.62 \mathrm{~g} / \mathrm{d}$ & 154 & 1.24 & $0.98,1.58$ & 1.08 & $0.85,1.38$ & $1 \cdot 10$ & $0.86,1.40$ & 0.98 & $0.75,1.27$ \\
\hline $1.62-1.90 \mathrm{~g} / \mathrm{d}$ & 199 & 1.44 & $1.14,1.83$ & $1 \cdot 14$ & $0.91,1.45$ & $1 \cdot 18$ & $0.93,1.49$ & 0.99 & $0 \cdot 76,1.30$ \\
\hline $1.90-2.27 \mathrm{~g} / \mathrm{d}$ & 228 & 1.50 & $1.18,1.90$ & $1 \cdot 10$ & $0.87,1.40$ & 1.14 & $0.90,1.45$ & 0.92 & $0.69,1.23$ \\
\hline$>2.27 \mathrm{~g} / \mathrm{d}$ & 253 & 1.62 & $1.27,2.05$ & 1.05 & $0.83,1.34$ & $1 \cdot 11$ & $0.87,1.41$ & 0.88 & $0.65,1.20$ \\
\hline
\end{tabular}

* Statistical analyses were conducted using Cox proportional hazard regression. All models were adjusted for sex by allowing baseline hazards among men and women to differ. † Model $1 \mathrm{~A}$ included baseline age.

$\ddagger$ Model $1 \mathrm{~B}$ included the variables of model $1 \mathrm{~A}$ and the following risk factors for peripheral artery disease: length of schooling, smoking, physical activity, waist circumference, BMI and alcohol intake.

$\S$ Model 2 included the variables of model 1B and the following potential intermediate variables: self-reported history of hypercholesterolaemia and/or use of lipid-lowering medication, hypertension and/or use of antihypertensive medication and diabetes mellitus and/or use of insulin.

\| Model 3 included the variables of model 1B and the following potential dietary risk factors: total energy intake, intake of fibre, glycaemic load, and intake of SFA, MUFA, linoleic acid and long-chain $n$-3 PUFA.

diagnoses of PAD were established and validated independent of the dietary assessment.

Identification of PAD cases in the present study relied on registered discharge diagnosis of PAD and the vast majority of identified cases were symptomatic patients. PAD may be underdiagnosed in the general population ${ }^{(40)}$ and cases either asymptomatic or not referred to hospitals were not included in the present study. However, random misclassification of a diagnosis of PAD may bias associations towards no association, but because PAD risk in general was relatively low, such potential bias was probably minor. We included detailed information on established risk factors for PAD in the analyses, but residual confounding from known or unknown PAD risk factors may still be of importance for the observed associations. The observed association between ALA intake and the rate of PAD (model 1A) was weakened after adjustment for established risk factors for PAD (model 1B). However, additional adjustment for history of hypercholesterolaemia, hypertension and diabetes (model 2) showed a similar pattern of association compared with model $1 \mathrm{~B}$, which may indicate that potential residual confounding from these co-morbidities was not of major importance. Notably, these co-morbidities may also be considered intermediates 
and conditioning for these covariates could potentially introduce collider stratification bias ${ }^{(39)}$, which may bias associations in either direction. In analyses including adjustment for established PAD risk factors and dietary factors (model 3) that may influence PAD risk, the observed association between ALA intake and the rate of PAD was slightly lower compared with model $1 \mathrm{~B}$ at higher ALA intakes and residual confounding from dietary factors cannot be excluded. However, adjustment for dietary factors may introduce restrictions in the underlying dietary pattern that are not comparable with the ordinary dietary pattern and analyses with and without dietary covariates should not be directly compared. Therefore, given the interpretational challenges of models 2 and 3, we consider model $1 \mathrm{~B}$ as the most appropriate for interpretation.

The Diet, Cancer and Health cohort only included native Danish participants from selected areas in Denmark who had survived until enrolment into the study without a previous diagnosis of cancer, chronic kidney disease or PAD, which may limit the generalisability of the study results.

We decided to conduct the main analyses as sex-stratified analyses by allowing baseline hazards among men and women to differ ${ }^{(5,6)}$. Thus, the hazard ratios from these analyses should be interpreted as a weighted average of the association in men and women. Previous studies have suggested that the endogenous conversion efficiency of ALA into LC $n$ - 3 PUFA may be stimulated by sex hormones and is greater in women ${ }^{(10)}$. However, in sex-specific analyses, we found similar patterns of association between ALA intake and the risk of PAD among men and women. Almost $60 \%$ of the female participants were post-menopausal at baseline ${ }^{(21)}$ and a potential higher conversion efficiency of ALA mediated by female sex hormones may therefore not be of major importance in this cohort. In supplemental analyses, additional adjustment for hormone substitution at baseline also did not influence the observed associations.

A previous cross-sectional study including 422 cases reported that ALA intake was associated with a lower odds of PAD ${ }^{(41)}$. Another cross-sectional study including 199 cases reported that the content of ALA in erythrocytes was associated with lower odds of lower limb disease ${ }^{(42)}$, whereas two case-control studies did not find any appreciably nor statistically significant differences between circulating levels of ALA between cases and controls ${ }^{(43,44)}$. However, none of these studies included detailed adjustment for risk factors of PAD and the results should be interpreted with caution due to the risk of residual confounding and reverse causation (cross-sectional studies)

We used restricted cubic splines to evaluate the shape of the association between ALA intake and the rate of PAD. ALA can be further metabolised into LC $n$-3 PUFA and lipid signalling molecules, which occurs in a complex biological pathway by enzymes that may be influenced by a combination of several factors including sex, genetics and background diet $^{(45)}$ that potentially could influence disease risk in a non-linear manner.

Previous studies have suggested that high intakes of the major $n$-6 PUFA linoleic acid and LC $n$ - 3 PUFA may lower the conversion efficiency of ALA into LC $n-3$ PUFA due to inhibition on shared enzymes. The median intake of LC $n-3$ PUFA in this cohort was $0.7 \mathrm{~g} / \mathrm{d}$, which was higher than that in cohort studies reporting inverse associations between ALA intake and
CHD $^{(15,16,18-20)}$ and this may be of importance for our study findings because a large study has suggested that ALA may reduce CHD risk in particular when intake of LC $n-3$ PUFA is low ${ }^{(17)}$. However, further well-powered studies investigating the role of genetics and intake of LC $n-3$ and $n-6$ PUFA on the association between ALA and the risk of atherosclerotic CVD are warranted.

In conclusion, dietary intake of ALA was not consistently associated with the risk of incident PAD among Danish middleaged men and women.

\section{Supplementary material}

For supplementary material/s referred to in this article, please visit https://doi.org/10.1017/S0007114519000874

\section{Acknowledgement}

The Danish Cancer Society funded the Diet, Cancer and Health study. The current study has been financially supported by the Danish Heart Foundation (CSB, 17-R115-A7415-22060), Helene and Georg Jensens and Ethel Merethe and Christian Pontoppidan's Fund. The funding agencies had no role in the design, analysis or writing of this manuscript.

All authors contributed to the conceptualisation of the present study. C. S. B. conducted the statistical analyses, prepared the tables and figures and wrote the first draft of the manuscript. C. S. B., A. N. L., S. L. C., M. U. J., P. C. C., E. B. S. and K. O. contributed to the planning of the statistical analyses, interpretation of the data and writing of the manuscript. S. L. C. supervised the conduct of the statistical analyses. A. T. contributed to the interpretation of the data and writing of the manuscript. All authors have read and approved the final manuscript.

There are no conflicts of interest.

\section{References}

1. Hiatt WR, Goldstone J, Smith SJ Jr, et al. (2008) Atherosclerotic peripheral vascular disease symposium II: nomenclature for vascular diseases. Circulation 118, 2826-2829.

2. Aboyans V, Ricco JB, Bartelink MEL, et al. (2018) 2017 ESC guidelines on the diagnosis and treatment of peripheral arterial diseases, in collaboration with the European Society for Vascular Surgery (ESVS). Eur Heart J 39, 763-821.

3. Patel MR, Conte MS, Cutlip DE, et al. (2015) Evaluation and treatment of patients with lower extremity peripheral artery disease: Consensus definitions from Peripheral Academic Research Consortium (PARC). J Am Coll Cardiol 65, 931-941.

4. Steg PG, Bhatt DL, Wilson PW, et al. (2007) One-year cardiovascular event rates in outpatients with atherothrombosis. JAMA 297, 1197-1206.

5. Fowkes FG, Aboyans V, Fowkes FJ, et al. (2017) Peripheral artery disease: epidemiology and global perspectives. Nat Rev Cardiol 14, 156-170.

6. Fowkes FG, Rudan D, Rudan I, et al. (2013) Comparison of global estimates of prevalence and risk factors for peripheral artery disease in 2000 and 2010: A systematic review and analysis. Lancet 382, 1329-1340. 
7. Calder PC (2015) Marine omega-3 fatty acids and inflammatory processes: effects, mechanisms and clinical relevance. Biochim Biophys Acta 1851, 469-484

8. De Caterina R (2011) n-3 Fatty acids in cardiovascular disease. N Engl J Med 364, 2439-2450.

9. Chang CL \& Deckelbaum RJ (2013) Omega-3 fatty acids: Mechanisms underlying 'protective effects' in atherosclerosis. Curr Opin Lipidol 24, 345-350.

10. Baker EJ, Miles EA, Burdge GC, et al. (2016) Metabolism and functional effects of plant-derived omega-3 fatty acids in humans. Prog Lipid Res 64, 30-56.

11. Rajaram S (2014) Health benefits of plant-derived alpha-linolenic acid. Am J Clin Nutr 100, 443-448.

12. de Lorgeril M \& Salen $\mathrm{P}$ (2007) Mediterranean diet and $n$-3 fatty acids in the prevention and treatment of cardiovascular disease. $J$ Cardiovasc Med 8, Suppl. 1, 38-41.

13. Estruch R, Ros E, Salas-Salvadó J, et al. (2018) Primary prevention of cardiovascular disease with a Mediterranean diet supplemented with extra-virgin olive oil or nuts. $N$ Engl J Med 378, e34.

14. Ruiz-Canela M, Estruch R, Corella D, et al. (2014) Association of Mediterranean diet with peripheral artery disease: The PREDIMED randomized trial. JAMA 311, 415-417.

15. Hu FB, Stampfer MJ, Manson JE, et al. (1999) Dietary intake of alpha-linolenic acid and risk of fatal ischemic heart disease among women. Am J Clin Nutr 69, 890-897.

16. Ascherio A, Rimm EB, Giovannucci EL, et al. (1996) Dietary fat and risk of coronary heart disease in men: cohort follow up study in the United States. BMJ 313, 84-90.

17. Mozaffarian D, Ascherio A, Hu FB, et al. (2005) Interplay between different polyunsaturated fatty acids and risk of coronary heart disease in men. Circulation 111, 157-164.

18. Vedtofte M, Jakobsen M, Lauritzen L, et al. (2014) Association between the intake of alpha-linolenic acid and the risk of CHD. Br J Nutr 112, 735-743.

19. Dolecek TA (1992) Epidemiological evidence of relationships between dietary polyunsaturated fatty acids and mortality in the multiple risk factor intervention trial. Proc Soc Exp Biol Med 200, 177-182.

20. Koh AS, Pan A, Wang R, et al. (2015) The association between dietary omega-3 fatty acids and cardiovascular death: the Singapore Chinese Health Study. Eur J Prev Cardiol 22, 364-372.

21. Bork CS, Jakobsen MU, Lundbye-Christensen S, et al. (2016) Dietary intake and adipose tissue content of alpha-linolenic acid and risk of myocardial infarction: a Danish cohort study. Am J Clin Nutr 104, 41-48.

22. de Goede J, Verschuren WM, Boer JM, et al. (2011) Alphalinolenic acid intake and 10-year incidence of coronary heart disease and stroke in 20000 middle-aged men and women in the Netherlands. PLOS ONE 6, e17967.

23. Fretts AM, Mozaffarian D, Siscovick DS, et al. (2014) Plasma phospholipid and dietary $\alpha$-linolenic acid, mortality, CHD and stroke: the Cardiovascular Health Study. Br J Nutr 112, 1206-2013.

24. Albert CM, Oh K, Whang W, et al. (2005) Dietary alpha-linolenic acid intake and risk of sudden cardiac death and coronary heart disease. Circulation 112, 3232-3238.

25. Oomen CM, Ocké M, Feskens EJ, et al. (2001) Alpha-linolenic acid intake is not beneficially associated with $10-\mathrm{y}$ risk of coronary artery disease incidence: the Zutphen Elderly Study. Am J Clin Nutr 74, 457-463.

26. Pietinen $P$, Ascherio A, Korhonen $P$, et al. (1997) Intake of fatty acids and risk of coronary heart disease in a cohort of Finnish men. The Alpha-Tocopherol, Beta-Carotene Cancer Prevention Study. Am J Epidemiol 145, 876-887.

27. Vedtofte MS, Jakobsen MU, Lauritzen L, et al. (2011) Dietary $\alpha$-linolenic acid, linoleic acid, and $n$ - 3 long-chain PUFA and risk of ischemic heart disease. Am J Clin Nutr 94, 1097-1103.

28. Rhee JJ, Kim E, Buring JE, et al. (2017) Fish consumption, omega-3 fatty acids, and risk of cardiovascular disease. Am J Prev Med 52, 10-19.

29. Larsson S, Virtamo J \& Wolk A (2012) Dietary fats and dietary cholesterol and risk of stroke in women. Atherosclerosis 221, 282-286.

30. Bork CS, Venø SK, Lundbye-Christensen S, et al. (2018) Dietary intake of $\alpha$-linolenic acid is not appreciably associated with risk of ischemic stroke among middle-aged Danish men and women. J Nutr 148, 952-958.

31. Tjønneland A, Olsen A, Boll K, et al. (2007) Study design, exposure variables, and socioeconomic determinants of participation in Diet, Cancer and Health: a population-based prospective cohort study of 57,053 men and women in Denmark. Scand J Public Health 35, 432-441.

32. Overvad K, Tjønneland A, Haraldsdóttir J, et al. (1991) Development of a semiquantitative food frequency questionnaire to assess food, energy and nutrient intake in Denmark. Int J Epidemiol 20, 900-905.

33. Tjønneland A, Overvad K, Haraldsdóttir J, et al. (1991) Validations of a semiquantitative food frequency questionnaire developed in Denmark. Int J Epidemiol 20, 906-912.

34. Willett W \& Stampfer MJ (1986) Total energy intake: implications for epidemiologic analyses. Am J Epidemiol 124, $17-27$.

35. Andersen TF, Madsen M, Jørgensen J, et al. (1996) The Danish National Hospital Register. A valuable source of data for modern health sciences. Dan Med Bull 46, 263-268.

36. Lynge E, Sandegaard JL \& Rebolj M (2011) The Danish national patient register. Scand J Public Health 39, 30-33.

37. Lasota AN, Overvad K, Eriksen HH, et al. (2017) Validity of peripheral arterial disease diagnoses in the Danish National Patient Registry. Eur J Vasc Endovasc Surg 53, 679-685.

38. Harrell FE (2015) Regression Modeling Strategies with Applications to Linear Models, Logistic and Ordinal Regression, and Survival Analysis, 2nd ed. New York: Springer.

39. Rothman K, Greenland S \& Lash T (2012) Modern Epidemiology, 3rd ed. Philadelphia, PA: Lippincott Williams and Wilkins.

40. Hirsch AT, Criqui MH, Treat-Jacobson D, et al. (2001) Peripheral arterial disease detection, awareness, and treatment in primary care. JAMA 286, 1317-1324.

41. Lane JS, Magno CP, Lane KT, et al. (2008) Nutrition impacts the prevalence of peripheral arterial disease in the United States. $J$ Vasc Surg 48, 897-904.

42. Leng GC, Taylor GS, Lee AJ, et al. (1999) Essential fatty acids and cardiovascular disease: the Edinburgh Artery Study. Vasc Med 4, 219-226.

43. Leng GC, Horrobin DF, Fowkes FG, et al. (1994) Plasma essential fatty acids, cigarette smoking, and dietary antioxidants in peripheral arterial disease. A population-based case-control study. Arterioscler Thromb 14, 471-478.

44. Gautam M, Izawa A, Shiba Y, et al. (2014) Importance of fatty acid compositions in patients with peripheral arterial disease. PLOS ONE 9, e107003.

45. Hodson L, Skeaff CM \& Fielding BA (2008) Fatty acid composition of adipose tissue and blood in humans and its use as a biomarker of dietary intake. Prog Lipid Res 47, 348-380. 